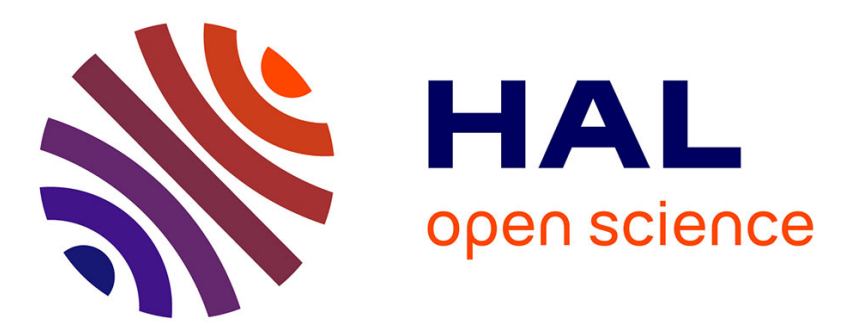

\title{
Quelles références aux pratiques de savoir en classe d'histoire? Eléments de réponses au texte de Sylvain Doussot
}

\author{
Didier Cariou
}

\section{- To cite this version:}

Didier Cariou. Quelles références aux pratiques de savoir en classe d'histoire? Eléments de réponses au texte de Sylvain Doussot. Revue Française de Pédagogie, 2018, 204, pp.73-80. hal-02424619

\section{HAL Id: hal-02424619 \\ https://hal.univ-brest.fr/hal-02424619}

Submitted on 27 Dec 2019

HAL is a multi-disciplinary open access archive for the deposit and dissemination of scientific research documents, whether they are published or not. The documents may come from teaching and research institutions in France or abroad, or from public or private research centers.
L'archive ouverte pluridisciplinaire HAL, est destinée au dépôt et à la diffusion de documents scientifiques de niveau recherche, publiés ou non, émanant des établissements d'enseignement et de recherche français ou étrangers, des laboratoires publics ou privés. 


\title{
Quelles références aux pratiques de savoir en classe d'histoire ? Éléments de réponses au texte de Sylvain Doussot
}

\author{
What references to knowledge practices in history class? Some answers to Sylvain \\ Doussot's text
}

\author{
Didier Cariou \\ Univ-Brest, CREAD EA 3875 \\ didier.cariou@univ-brest.fr
}

Cet article propose des réponses à l'article de Sylvain Doussot publié dans ce numéro à propos d'un article précédent paru dans le numéro 197 de la Revue française de pédagogie. Ce dernier proposait une analyse des modalités de lecture d'un document historique par des élèves. Sur un plan épistémologique, notre débat renvoie aux pratiques de lecture des historiens méthodiques et des historiens des Annales. Les premiers construisaient le fait historique à partir des documents. Les seconds posaient d'abord un problème et le document servait à apporter une réponse à ce problème. Sur le plan didactique, le rappel de cette différence conduit à réévaluer les pratiques de lecture des élèves étudiées dans l'article initial : elles sembleraient plus proches de celles des historiens des Annales. Ce débat conduit à la question du rapport entre les pratiques de classe et les pratiques savantes des historiens. Nos cadres respectifs de la Problématisation et de la Théorie de l'action conjointe en didactique (TACD) proposent des réponses différentes à cette question.

Mots-clés (TESE) : didactique, histoire, processus d'apprentissage, enseignement

This article provides answers to Sylvain Doussot's article published in this issue about a previous article published in the issue 197 of the Revue française de pédagogie. It provided an analysis of how students read a historical document. From an epistemological point of view, our debate refers to the reading practices of methodical historians and historians of the Annales. The former built the historical fact from the documents. The latter raised first of all a problem and the document was used to provide an answer to this problem. From a didactic point of view, the reminder of this difference leads to a reassessment of the reading practices of the students studied in the former article: they would seem closer to those of the historians of the Annales. This debate leads to the question of the relationship between classroom practices and scholarly practices of historians. Our respective frameworks, the Problematization framework and the joint action theory in didactics (TACD) framework, produce different answers to this question.

Keywords (TESE): didactics, history, learning process, teaching

Je remercie les rédacteurs en chef de la Revue française de pédagogie de m’offrir l'opportunité de répondre à l'article de Sylvain Doussot qui questionne mon propre article (Cariou, 2016). Ils nous permettent de poursuivre un débat engagé il y a plusieurs années déjà (Cariou, 2013a, 2013b ; Doussot, 2013). Bien entendu ce débat ne présente d'intérêt que parce que, au-delà de nos articles respectifs dans le champ très circonscrit de la didactique de l'histoire, il questionne les relations entre deux cadres théoriques parents mais distincts, la Problématisation et la Théorie de l'action conjointe en didactique (TACD) (Sensevy, 2011 ; Collectif DPE, 2019), dans le champ des recherches comparatistes en didactique. 
Je distingue deux points dans le texte de Sylvain Doussot pour structurer ma réponse. Le premier porte sur les différences épistémologiques entre le courant de l'école méthodique de la fin du XIXe siècle et celui des Annales irriguant la pratique historienne depuis le milieu du XXe siècle. Ces différences fondent la distinction que j'opérais dans mon article entre paradigme informatif et paradigme indiciaire qu'il convient effectivement de questionner. Le second point, qui en découle, concerne la comparaison entre les pratiques de savoir des historiens et les pratiques des élèves et du professeur en classe d'histoire ainsi que la façon dont la TACD propose une théorie de la pratique par le « modèle du jeu » (Sensevy, 2012).

\section{Deux paradigmes historiques distincts?}

Un premier point concerne le questionnement de Sylvain Doussot (p. 59-60) au sujet de la séparation que j'introduis entre, d'une part un paradigme scientiste et objectiviste assigné à l'historien Fustel de Coulanges, et d'autre part un paradigme que j'ai caractérisé comme indiciaire et regroupant les historiens méthodiques (Langlois et Seignobos), les historiens des Annales (Marc Bloch et Lucien Febvre) ainsi que Ginzburg.

\section{La dichotomie méthodologique de l'école méthodique}

Ce questionnement m'a engagé dans une relecture des ouvrages de Langlois et Seignobos (1898) d'une part, de Bloch (1949 et de Febvre (1953) d'autre part, pour revenir sur la différence effectivement fondamentale entre l'épistémologie des historiens méthodiques et des historiens du courant des Annales.

À l'époque des historiens méthodiques, le scientifique des sciences de la nature pensait pouvoir observer directement son objet d'étude, en utilisant au besoin un microscope ou un télescope. Dans la même logique, selon le manuel rédigé par Langlois et Seignobos (1898) en direction des étudiants en histoire, la science historique procéderait en deux temps dissociés : l'établissement des faits par l'historien à partir de la critique externe et interne des témoignages et des documents puis le questionnement de ces faits en vue de les interpréter, de les catégoriser et d'expliquer leurs enchaînements afin de produire un discours intelligible sur le passé. La dimension véritablement scientifique de l'histoire se tiendrait dans le passage de l'établissement des faits à leur interprétation (Seignobos, 1901, p. 93). Toutefois, les faits historiques ne seraient pas directement accessibles par la seule lecture des documents. Pour être compris, ces faits révolus devraient être imaginés à partir des documents par les historiens qui s'en donnent une représentation mentale par analogie avec des faits du passé déjà connus d'eux ou avec des situations sociales qu'ils ont euxmêmes vécues (Langlois \& Seignobos, 1898, p. 181-187 ; Seignobos, 1901, p. 115-124). En d'autres termes, les faits historiques seraient établis par l'analyse critique des documents qui permettrait de constituer les affirmations des documents en faits historiques, à l'instar des pratiques scientifiques de l'époque. Ils seraient ensuite construits par tri, regroupement, catégorisation et interprétation (Seignobos, 1901, p. 94-109).

Or, la dichotomie entre l'établissement des faits et leur construction n'est pas tenable. Elle suppose que les documents et les faits soient premiers dans l'enquête de l'historien. En réalité, tout historien mobilise des documents et des faits à partir des questions qu'il pose au passé et de l'argumentation sur la base de laquelle il choisit, construit et interprète ces faits (Prost, 1996, p. 7376).

Si Langlois et Seignobos défendaient malgré tout cette dichotomie, c'est sans doute aussi dans un souci d'exposition de la méthode historique aux étudiants auxquels leur manuel était destiné. De même, ils considéraient que les professeurs de l'enseignement secondaire devaient d'abord présenter les faits historiques pour que les élèves puissent s'en faire une représentation, avant d'en venir à leur interprétation. C'est pourquoi leur " épistémologie est une didactique » (Prost, 1994, p. 115). Ou une « épistémologie de manuel » (Doussot, p. 62). Cette dichotomie ne nous renseignerait pas sur les pratiques effectives des historiens, elle montrerait plutôt comment rendre l'histoire enseignable. Elle fonderait encore aujourd'hui la forme scolaire de l'histoire et le modèle de la boucle didactique (Audigier, Cremieux \& Mousseau, 1996) : le professeur questionne 
les élèves sur les documents supports de la séance, les élèves énoncent des réponses souvent partielles qui sont validées ou non par le professeur qui produit ensuite des explications et généralise à partir des constats des élèves. Et ainsi de suite, selon une conception qui tendrait à considérer que les réponses aux questions du professeur sont " dans » les documents. Les faits seraient établis lors de la lecture des documents et construits ensuite par l'interprétation du professeur. Il est donc effectivement risqué de prendre au pied de la lettre le propos du manuel de Langlois et Seignobos (1898) et de le considérer comme une présentation de la pratique effective des historiens. Il nous renseignerait davantage sur les pratiques de classe que sur les pratiques des historiens.

\section{L'épistémologie du problème du courant des Annales}

De leur côté, Bloch et Febvre ont pointé l'influence du développement de la microbiologie, de la physique quantique et de la théorie de la relativité sur leur propre démarche (Oexle, 1990 ; Bloch, 1949, p. 45-46 ; Febvre, 1953, p. 28-31). Ces sciences ne supposent plus nécessairement l'observation du réel mais procèdent notamment de raisonnements mathématiques destinés à répondre aux problèmes que se posent les scientifiques et dont les solutions n’ont parfois été vérifiées empiriquement qu'au bout de plusieurs décennies. Elles se fondent également sur l'utilisation d'instruments de plus en plus complexes qui rendent possibles ces raisonnements mathématiques. En histoire comme en physique, le statut de l'observation et des énoncés d'observation s'en est trouvé modifié.

Il en résulte que l'on peut voir toutes les sciences comme procédant de la construction d'un problème qui fonde des hypothèses vérifiées par leur mise à l'épreuve du réel. Febvre désigne cette démarche problématisée comme une " étude scientifiquement conduite " à partir d'un problème (Febvre, 1953, p. 22). C'est pourquoi, à l'affirmation ouvrant le premier chapitre de l'ouvrage de Langlois et Seignobos (1898, p. 29) : " Pas de documents, pas d'histoire », Febvre (1953 p. 22) rétorquait : " Pas de problèmes, pas d'histoire ». Selon Bloch et Febvre, les documents ne parlent pas d'eux-mêmes pour fournir un accès aux faits du passé, ils " ne parlent que lorsqu'on sait les interroger », car : " toute recherche historique suppose, dès ses premiers pas, que l'enquête ait déjà une direction » (Bloch, 1949, p. 77 ; Doussot, p. 61-62). Certes, Langlois et Seignobos (1898, p. 177-179) expliquaient que l'historien, à l'instar de tous les scientifiques, devait poser des questions. Cependant, ils posaient ces questions non pas aux documents mais aux faits déjà établis, afin de les construire par une catégorisation et une explication. De même, les pratiques habituelles de classe partent des documents et pas d'un problème historique. Leur référence savante ne peut donc se trouver que du côté de l'école méthodique.

Tous ces historiens partageaient cependant la conception du document historique comme une " trace » du passé qui subsiste dans le présent, et de l’histoire comme une " connaissance par traces »(Bloch, 1949, p. 71 ; Langlois \& Seignobos, 1898, p. 65-66). Cependant, Langlois et Seignobos n'en tirèrent aucune conclusion méthodologique - selon eux, la trace était constituée des affirmations des documents à partir desquelles l'historien établit les faits du passé - alors que la notion de trace fonde le paradigme indiciaire mis en évidence par Ginzburg (1986). Dans cette conception, un texte, un document iconographique ou un fragment de poterie venus du passé sont érigés en traces par l'historien quand ce dernier considère que l'un de ces objets lui permet de mener une enquête sur le problème qui se pose à lui. La trace fournit des indices à partir desquels l'historien produit des conjectures sur les faits du passé qu'il étudie dans le cadre d'un problème pour atteindre, par ce raisonnement ou cette enquête, une connaissance de ces faits en fonction des questions qu'il se pose et qu'il pose à ces traces.

En résumé, nous retenons des écrits de Bloch, Febvre et Ginzburg que la pratique historienne consiste en une enquête qui procède d'un problème. Ce problème conduit l'historien à énoncer des hypothèses qu'il vérifie par la critique des documents considérés comme des traces du passé. Ces traces fournissent des indices que l'historien analyse pour établir des conjectures sur les faits du passé. On le voit, cette rapide catégorisation de la pratique historienne emprunte essentiellement aux analyses de Bloch, Febvre et Ginzburg. Elle laisse de côté les analyses de 
Langlois et Seignobos et, contrairement à ce que j'affirmais dans mon article, rend moins centrales la critique interne et la critique externe des documents.

\section{Jeux d'apprentissage et jeux épistémiques en classe d’histoire} À quel jeu jouent les élèves?

En quoi ce retour aux courants historiographiques peut-il servir à rendre compte des stratégies des élèves de CM1 et de quatrième face à l'image révolutionnaire de 1789 qui leur est donnée à étudier ? Pour répondre à cette question, je reviens sur le passage de mon article (Cariou, 2016, p. 72) concernant l'interrogation des élèves de la classe de CM1 au sujet de l'identité des deux personnages juchés sur le dos du paysan. L’un des deux personnages porte une épée au côté, et certains élèves l'assimilent au roi qui portait également une épée au côté, ainsi qu'ils l'avaient constaté en étudiant la semaine précédente le portrait de Louis XIV en costume de sacre. Je considérais qu'ils voyaient l'épée comme une information sur le passé fournie directement par le document : cette épée est l'épée du roi. En revanche, l'élève que nous nommons Emmanuel voyait cette épée comme un indice à interpréter : elle est autre chose que ce qu'elle semble être sur l'image et elle signale l'appartenance à la noblesse.

Contrairement à ce que j'énonce dans mon article, Sylvain Doussot (p. 66-68) propose de considérer que tous les élèves s'engagent de fait dans une pratique critique qui les conduit à sélectionner une information fournie par le document et à l'articuler à un savoir déjà-là sur la période. Emmanuel relierait l'épée du noble au statut de la noblesse sous l'Ancien Régime. Natacha, Hélène et Mélina relieraient l'objet porté autour du cou par ce même noble (« un collier avec un soleil ») à celui du Roi-Soleil lui-même. Là où je verrais un jeu d'apprentissage gagnant pour Emmanuel qui distingue le noble sur l'image et un jeu de devinette perdant pour ses camarades qui confondent le personnage du noble et celui du roi, Sylvain Doussot considère que tous jouent le jeu de la critique des sources. Par des énoncés modalisés, les élèves proposeraient des interprétations sur l'identité des personnages de l'image. Ils s'engageraient dans une controverse destinée à explorer le degré de possibilité des propositions qui s'appuient sur des indices (l'épée et le collier sur l'image) afin de proposer une conjecture sur l'identité du personnage du noble. Cette controverse procéderait d'une tension entre les faits ou les données (l'épée et le collier sur l'image, le roi, les guerriers et les nobles à l'époque) et des registres explicatifs (le roi est au-dessus des nobles, etc.). Sylvain Doussot explique qu'il ne s'agit alors pas d'analyser les propositions des élèves en termes de vérité ou d'erreur, mais de les considérer comme une exploration des possibles parmi les interprétations proposées afin de retenir les explications qui semblent fondées. Nous retrouvons ici l'espace des contraintes développé en Problématisation à la suite des travaux de Orange (2005) et dans le cadre duquel s'effectue l'exploration d'un problème par la mise en tension du registre empirique des données, du registre des contraintes et du registre explicatif des modèles (Doussot, 2018). Les échanges entre les élèves seraient gros d'une pratique de savoir potentielle, celle d'une enquête sur les conditions de validité de leurs énoncés dans le cadre d'une controverse fondée sur un problème, où il s'agirait moins « de chercher la réponse » que « de re-chercher la valeur de la réponse en en faisant l'objet de l'enquête » (Doussot, p. 69). L'accès à cette controverse supposerait que le professeur intervienne non pas pour valider ou invalider les réponses des élèves à partir d'une position dominante, mais pour accompagner les élèves en rappelant les règles du jeu ou en exprimant les stratégies possibles pour s'engager dans l'exploration d'un problème.

Cette objection de Sylvain Doussot vaut que l'on s'y attarde. Rétrospectivement, il me semble effectivement moins pertinent d'instituer une différence entre la démarche d'Emmanuel et celle de ses camarades. On peut effectivement considérer que les élèves se trouvent face à un problème de lecture de l'image (qui sont les personnages assis sur le dos du paysan ?) qui est l'enjeu du jeu d'apprentissage. Les élèves avancent, produisent des conjectures sur les indices produits par l'image en se référant à des savoirs déjà acquis. En d'autres termes, ils explorent les formes sémiotiques du milieu qui posent problème en s'appuyant sur le savoir déjà-là dans le contrat didactique. À la différence des «situations forcées » de la Problématisation, la professeure intervient peu, elle est réticente, et se contente de relancer les échanges entre les élèves. Elle 
intervient finalement pour invalider une conjecture erronée qui assimile le personnage du noble à celui du Roi-Soleil. Dans ce passage, les élèves s'engagent de fait dans une démarche indiciaire de production de conjectures à propos de la signification de l'épée et du collier portés par le personnage du noble.

\section{Le modèle du jeu en TACD}

Pour développer la suite de mon propos, je me réfère à une étude menée conjointement par un chercheur se réclamant de la TACD et une chercheuse se réclamant de la Problématisation (Santini \& Crépin-Obert, 2015). Cette étude montre que le cadre de la Problématisation promeut la participation des élèves à la construction d'un problème et à l'enquête sur ce problème. L'examen des arguments produits par les élèves vise l'établissement du caractère apodictique des savoirs construits tout au long de l'enquête des élèves. Pour la TACD, le savoir est envisagé en tant que pratique de savoir potentiellement dotée d'une certaine efficacité en situation. Elle est susceptible de développer la puissance d'agir des agents afin de résoudre le problème qui se pose à eux. La description de la grammaire de l'action effective, au sens de Wittgenstein (1953 § 90), permet d'envisager la façon dont l'action conjointe du professeur et des élèves exprime ou non cette puissance d'agir. Elle conduit à voir une séance de classe comme un ensemble de jeux de langage (Wittgenstein, 1953) et à modéliser l'action didactique comme un ensemble de jeux d'apprentissage. Bien entendu, du côté de la TACD, il est mis en avant que l'élève ne peut développer son pouvoir d'agir et construire des savoirs sans lien avec les problèmes qui les fondent. On ne peut pas détacher les jeux des problèmes qui en sont à l'origine et qui en constituent l'enjeu. Du côté de la Problématisation, il est souligné que l'élève ne peut travailler des problèmes sans développer son pouvoir d'agir. Dans mon article, je montre que les élèves des deux classes essaient surtout de faire quelque chose avec cette image révolutionnaire. À charge pour le chercheur d'essayer de comprendre à quoi ils jouent en décrivant le jeu auquel ils jouent (Wittgenstein, 1953, $\S 54$; repris par Bazin, 2008, p. 355). Il s’agit ici d’interpréter un document mais sans articuler l'interprétation à un problème historique. Pour les élèves, c'est l'accès à la signification de cette image grotesque qui constitue le problème.

C'est sans doute l'orientation de notre regard respectif sur ce qu'il y a à voir dans une séance d'apprentissage et sur le rapport entre les pratiques du monde scolaire et les pratiques du monde de la culture, qui organise " la normativité des deux approches » (Doussot, p. 58). En effet, ce qui prête surtout à controverse entre les chercheurs tenant de la Problématisation et de la TACD et que met bien en évidence le texte de Doussot - tient, me semble-t-il, à l'articulation entre les jeux d'apprentissage, modélisant l'action conjointe effective, ici et maintenant, du professeur et des élèves dans la classe, et les jeux épistémiques modélisant les pratiques culturelles que les connaisseurs - en l'occurrence les historiens - entretiennent avec un savoir (Sensevy, 2011 ; Collectif DPE, 2019). Selon la TACD, le jeu d'apprentissage dans la classe diffère du jeu épistémique dans le monde de la culture mais peut entretenir avec lui une parenté épistémique car certains éléments de la pratique du connaisseur peuvent se retrouver en acte dans la pratique de la classe modélisée comme un jeu d'apprentissage. En revanche, me semble-t-il, pour la Problématisation, la modélisation en terme de problème de ce qui se passe dans la classe est identique à la modélisation de la pratique des historiens.

Il me semble que c’est au cœur de la question de la parenté épistémique et de la façon de modéliser ce qui se passe dans la classe que se tient l'enjeu du débat présent. En effet, dans nos arrière-plans respectifs, se tient la continuité de l'enquête selon Dewey (1938). Mais la Problématisation articule la notion de continuité de l'enquête à celle de la rupture bachelardienne (Fabre, 2009). Du côté de la TACD, la notion de continuité de l'enquête nous conduit à essayer de voir s'il est possible, dans ce qu'il rend éventuellement l'élève capable de se livrer à une pratique de savoir, de comparer les capacités épistémiques dont le jeu d'apprentissage rend les élèves capables avec certains éléments d'un jeu épistémique. Ce que nous nommons un « jeu d'apprentissage gagnant » permet aux élèves de développer une capacité épistémique qui présente un " air de famille » avec certaines caractéristiques d’un jeu épistémique dans le monde de la culture et qui 
permet de s'approprier un savoir. Mais nous ne pensons pas que l'action du professeur et des élèves puisse être directement modélisée sous forme de jeux épistémiques car ces derniers rendent compte des pratiques de savoirs dans le monde de la culture et ne peuvent être envisagés que comme éléments de comparaison avec les jeux d'apprentissage. Les élèves ne jouent pas à l’identique le jeu épistémique de l’historien.

En revanche, il me semble que Doussot considère qu'il faudrait " passer " d'un jeu d'apprentissage à un jeu épistémique pour accéder à un « jeu de problématisation » (p. 68). Je me demande si, pour la Problématisation, la notion de continuité de l'enquête ne tiendrait pas dans le fait que la modélisation de l'enquête autour du problème rend compte aussi bien des pratiques des historiens que des pratiques dans la classe. La rupture tiendrait à ce que ces pratiques problématisées, celles des historiens comme celles des élèves, se trouveraient en rupture avec les pratiques habituelles de classe, telles que celles que j'étudie.

Cependant, dans notre débat, la disparité des références de nos cadres théoriques rend sans doute obscures les analyses produites par les tenants de l'autre cadre théorique. Ainsi, Sylvain Doussot évoque « le jeu d'apprentissage voulu par l'enseignant » et le « jeu d'apprentissage de la professeure » (p. 61). Or, le modèle du jeu d'apprentissage, selon la logique grammaticale dedescription de l'action, conduit à décrire et à modéliser non pas ce que " veut » l'enseignant mais, selon un postulat pragmatiste, ce que font effectivement et conjointement le professeur et les élèves au coeur de la dialectique du contrat didactique et du milieu. Même si les professeures sont réticentes à certains moments (Doussot, p. 67), cela ne signifie pas qu'elles sont absentes de l'action conjointe car la réticence n'est jamais exempte d'une part d'expression (Sensevy, 2011 ; Collectif DPE, 2019). Même si les professeures, en position haute, posent les questions et valident les réponses des élèves (Doussot, note 13, p. 69), elles s’impliquent malgré tout dans une action conjointe où les réponses des élèves découlent de l'analyse des attentes des professeures et où les questions posées par ces dernières découlent de l’analyse des réponses des élèves.

\section{Sens du jeu et règles du jeu}

Je suis d'accord sur le fait que la notion de contrat didactique - entendue au sens d'un habitus didactique constitué du déjà-là des savoirs et des capacités incorporées qui permet aux élèves d'interpréter les attentes du professeur à leur égard pour s'orienter parmi les formes sémiotiques du milieu didactique (Sensevy, 2011, p. 106) - se situe moins du côté de la notion de paradigme au sens de Kuhn (Doussot, note 11, p. 66) que de la notion de sens du jeu ou de sens pratique (Bourdieu, 1980). Mais la notion de sens du jeu renvoie d'abord au modèle du jeu d'apprentissage qui décrit la dialectique d'un contrat didactique et d'un milieu et qui ne s'en tient pas au seul contrat didactique. Le modèle du jeu montre comment s'incarne le sens du jeu. Il constitue une première description de la dialectique du contrat et du milieu qui fonde le sens du jeu des agents. Peut-on pour autant réduire la description du sens du jeu à la description des règles du jeu?

La dichotomie de l'établissement des faits et de leur interprétation relève certes des « règles demi-savantes », selon Bourdieu (1980, p. 176), établies comme nous l'avons vu par le manuel de Langlois et Seignobos (1898) (Doussot, p. 63). L'analyse que je propose des deux séances de lecture de l'image révolutionnaire à l'aune de cette dichotomie relèverait selon Sylvain Doussot d'un inconscient disciplinaire, au sens de Bourdieu, lié à ma trop grande proximité avec mon objet d'étude. Je suis certes un vieux professeur d'histoire-géographie avant d'être un chercheur en didactique. Mais là n’est pas la question, à mon avis. Pour la classe de sciences, Santini (2013, p. 72-74) a montré que le jeu d'apprentissage de la compréhension des séismes pouvait être comparé à des modèles géologiques élaborés par différentes générations de géologues. Or, les jeux d'apprentissage souvent joués dans les classes d'histoire relèvent d'abord de la forme " questionréponse-tâche » (Collectif DPE, 2019) par laquelle les élèves cherchent à interpréter les attentes de leur professeure afin de produire la réponse supposée attendue. C'est le contrat didactique habituel en classe d'histoire et c'est globalement ainsi que les professeures organisent la séance d'analyse de l’image révolutionnaire. Les pratiques habituelles en classe d'histoire, décrites dans mon article et 
formalisées dans les instructions officielles, renvoient également à la dichotomie de la critique et de l'explication au cœur de la linéarité suivante : présentation du document, recherche d'informations, explication. Elles ont quelque chose à voir avec la dichotomie de l'établissement et de la construction des faits promue par Langlois et Seignobos (1992).

Cependant, il arrive que la modélisation en termes de jeux d'apprentissage permette de signaler une capacité épistémique naissante, une stratégie embryonnaire manifestée par un ou plusieurs élèves qui s'affranchissent de règles du jeu explicites pour produire des conjectures. Cette stratégie pourrait être convertie par le professeur en une stratégie pour toute la classe qui aurait davantage de familiarité avec le jeu épistémique du connaisseur. Notre débat autour de la signification du passage où les élèves produisent des conjectures sur l'épée et le collier du personnage du noble alors que leur professeure reste relativement réticente signale une pratique de lecture du document qui gagnerait à être davantage systématisée.

\section{Conclusion}

Ce type de débat entre chercheurs travaillant sur des objets similaires tout en relevant de cadres théoriques différents permet de revenir sur certaines analyses et de repréciser certains éléments de la théorie. Pour conclure, je souhaite revenir sur les points soulevés dans la conclusion du texte de Sylvain Doussot.

Le premier point concerne la référence à la règle selon Bourdieu (1980, p. 174-176). Sylvain Doussot (p. 63) semble considérer que, par une forme de biais scolastique, la modélisation de la pratique en jeux d'apprentissage tendrait à plaquer les règles du jeu sur la pratique observée et à oublier que ces règles ne sont pas la réalité. Par la modélisation de la pratique de savoir sous la forme d'un jeu d'apprentissage et par le découpage de ce dernier en une série de jeux élémentaires du type : présentation des documents, prélèvement d'information, production d'explication -, les règles du jeu seraient considérées par moi comme les règles effectives de la pratique observée en classe d'histoire. Certes, le langage du modèle du jeu décrit les « règles du jeu » de la pratique que nous nommons en TACD les règles définitoires et les règles stratégiques du jeu d'apprentissage. Mais il convient de ne pas oublier qu'il s'agit du langage du modèle et pas de la réalité qu'il modélise. Je rappelle à cet égard la phrase de Bourdieu : " L'image du jeu est sans doute la moins mauvaise pour évoquer les choses sociales » (Bourdieu, 1987, p. 80). Dans la pratique, les agents n’appliquent pas des règles explicites qui auraient été édictées par un nomothète. Ils déploient des stratégies effectives et agissent comme il est habituel d'agir dans telle situation (Bourdieu, 1980, p. 111-113). Comme le rappelle Wittgenstein (1953, § 68), une règle du jeu au tennis est de lancer la balle au-dessus du filet. Mais aucune règle ne décide de la stratégie du joueur quand il décide de lancer la balle au ras du filet ou beaucoup plus haut. En classe d'histoire, ce ne sont pas les règles définitoires du jeu qui façonnent la pratique, il s'avère plutôt que, pour toute une série de raisons, les professeurs et les élèves agissent généralement de la façon que j’ai tenté de décrire et qui est difficilement comparable à celle de l'histoire-problème des Annales.

Le second point concerne également des perspectives de travail communes. Une perspective consiste à poursuivre ce travail d'intercompréhension de nos cadres théoriques respectifs qui contribue à mieux revenir sur nos propres analyses. La perspective qui en découle est de travailler conjointement à la transformation de la forme scolaire de l'histoire. Les analyses en TACD montrent pour quelles raisons les pratiques habituelles de classe produisent des apprentissages de faible intensité. Elles justifient la construction par Sylvain Doussot et ses collègues de situations de classe raisonnablement proches des pratiques savantes problématisées. Il s'agit de savoir maintenant à quelles conditions celles-ci peuvent être systématisées pour rapprocher davantage les pratiques de savoir des élèves de celles des historiens pour que l'histoire scolaire soit véritablement une discipline qui exerce l’intelligence des élèves. 


\section{Bibliographie}

AUDIGIER F., CREMIEUX C. \& MOUSSEAU M.-J. (1996). L'enseignement de l'histoire et de la géographie en troisième et en seconde. Étude descriptive et comparative. Paris : INRP.

BAZIN J. (2008). Des clous dans la Joconde. L'anthropologie autrement. Toulouse : Anacharsis.

BLOCH M. ([1949] 1993). Apologie pour l'histoire ou Métier d'historien. Paris : Armand Colin.

BOURDIEU P. (1980). Le sens pratique. Paris : Éd. de Minuit.

BOURDIEU P. (1987). Choses dites. Paris : Éd. de Minuit.

CARIOU D. (2013a). « Les déséquilibres entre contrat et milieu dans une séance d'histoire à l'école primaire. Une étude exploratoire ». Éducation et didactique, 7(1), p. 9-32.

CARIOU D. (2013b). « Comment construire un milieu et un jeu d'apprentissage gagnant en histoire ? Questions adressées à la problématisation en guise de réponse à Sylvain Doussot ». Éducation et didactique, no 7(3), p. 123-126.

CARIOU D. (2016). « Information ou indice ? Deux lectures d'une image en classe d'histoire ». Revue française de pédagogie, 197, p. 63-78.

COLLECTIF DPE (2019). Didactique pour enseigner. Rennes : Presses universitaires de Rennes.

DEWEY J. ([1938] 1967). Logique. La théorie de l'enquête. Paris : PUF.

DOUSSOT S. (2013). «Quels problèmes pour les jeux d'apprentissage ? Questions à partir d'un article de Didier Cariou en didactique de l'histoire ». Éducation et didactique, 7(3), p. 117-122.

DOUSSOT S. (2018). L'apprentissage de l'histoire par problématisation. Enquêter sur des cas exemplaires pour développer des savoirs et compétences critiques. Berne : Peter Lang.

FABRE M. (2009). Philosophie et pédagogie du problème. Paris : Vrin.

FEBVRE L. ([1953] 1992). Combats pour l'histoire. Paris : Pocket.

GINZBURG C. ([1986] 1989). " Traces. Racines d'un paradigme indiciaire ». In Mythes, emblèmes, traces. Morphologie et histoire. Paris : Flammarion, p. 139-180.

LANGLOIS C.-V. \& SEIGNOBOS C. ([1898] 1992). Introduction aux études historiques. Paris : Éd. Kimé.

OEXLE O. G. (1990). « Marc Bloch et la critique de la raison historique ». In H. Atsma \& A. Burguière (dir.), Marc Bloch aujourd'hui. Histoire comparée et sciences sociales. Paris : Éditions de l’École des Hautes Études, p. 419-433.

ORANGE C. (2005). «Problématisation et conceptualisation en sciences et dans les apprentissages scientifiques ». Les Sciences de l'éducation - Pour l'Ère nouvelle, 38(3), p. 69-94.

PROST A. (1994). « Charles Seignobos revisité ». Vingtième siècle. Revue d'histoire, 43(1), p. 100118.

PROST A. (1996). Douze leçons sur l'histoire. Paris : Éd. du Seuil.

SANTINI J. (2013). « Une étude du système de jeux de savoirs dans la Théorie de l'Action conjointe en Didactique. Le cas de l'usage des modèles concrets en géologie au Cours Moyen ». Éducation et didactique, 7(2), p. 69-94.

SANTINI J. \& CRÉPIN-OBERT P. (2015). « Analyse comparée de séances de géologie à l’école primaire. Problématisation et action conjointe élève-professeur ». Revue de didactique des sciences et techniques, 11, p. 25-78.

SEIGNOBOS C. ([1901] 2014). La méthode historique appliquée aux sciences sociales. Lyon : ENS Éditions.

SENSEVY G. (2011). Le sens du savoir : éléments pour une théorie de l'action conjointe en didactique. Bruxelles : De Boeck.

SENSEVY. G. (2012). « Le jeu comme modèle de l'activité humaine et comme modèle en théorie de l'action conjointe en didactique. Quelques remarques ». NPSS, 7(2), p. 105-132.

WITTGENSTEIN L. ([1953] 2004). Recherches philosophiques. Paris : Gallimard. 